\title{
Infrared Observations of Interstellar Ices
}

\author{
P. Ehrenfreund and W. A. Schutte \\ Raymond \& Beverly Sackler Laboratory for Astrophysics at Leiden \\ Observatory, P.O. Box 9513, 2300 RA Leiden, The Netherlands
}

\begin{abstract}
In the recent years revolutionary results concerning the nature of icy dust particles have been obtained with the help of the Infrared Space Observatory (ISO) and ground based observations. To date interstellar ice features of $\mathrm{H}_{2} \mathrm{O}, \mathrm{CO}, \mathrm{CO}_{2}, \mathrm{CH}_{3} \mathrm{OH}, \mathrm{CH}_{4}, \mathrm{H}_{2} \mathrm{CO}$, OCS and $\mathrm{HCOOH}$ as well as other minor species are observed. Interstellar grains act as important catalysts in the interstellar medium. Processes such as UV irradiation, cosmic ray processing and temperature variations determine the grain mantle growth and chemical evolution. ISO has revealed that ice segregation is an important and ubiquitous process in the vicinity of massive protostars and reflects the extensive thermal processing of grains in such environments.

In this paper a recent view on the inventory of interstellar ices is presented. Constraints on the reservoirs of oxygen in dense clouds are discussed, taking into account recent measurements of oxygen-bearing species. Large abundances of $\mathrm{CO}_{2}$ and $\mathrm{CH}_{3} \mathrm{OH}$ in dense molecular clouds provide challenging perspectives to investigate the differences of ice chemistry in the vicinity of high and low-mass protostars. Accurate abundances of ice species and knowledge on the ice distribution in the protostellar regions are an important tool to define the environmental conditions in molecular clouds. A global understanding of interstellar ice chemistry also allows monitoring the incorporation and evolution of volatiles in planetesimals and comets and to reveal processes predominant in the early Solar System.
\end{abstract}

\section{Introduction}

Icy grain mantles which cover dust particles in dense cold clouds have been revisited with the Infrared Space Observatory (ISO). ISO data in combination with high resolution ground based measurements allow us to reconstruct the environmental conditions and grain composition in star-forming regions. This review is aimed to show the current picture of interstellar ice chemistry and discusses most of the features visible in the infrared spectrum of dense clouds between $2.5-20 \mu \mathrm{m}$.

Icy grain mantles are formed in cold, dense environments by efficient accretion of atoms and molecules from the interstellar gas. Diffusion of small species allows reaction pathways on the grain surface, leading to the formation of new molecules. Regulatory mechanisms such as selective desorption, subli- 


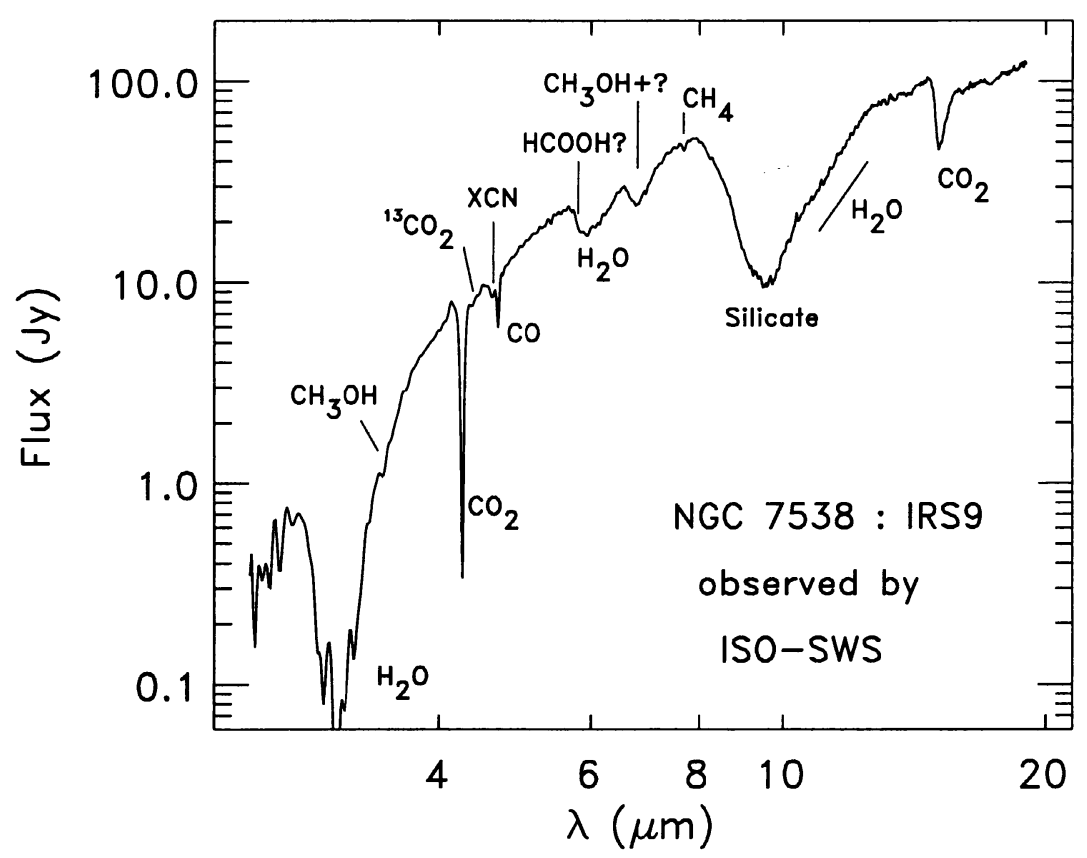

Figure 1. The ISO-SWS spectrum toward the massive protostar NGC7538:IRS9 between 2.5-20 $\mu \mathrm{m}$ (Whittet et al. 1996).

mation and grain explosion desorb material and return molecules back in the gas phase. The evolution of interstellar ices is determined by processes in the protostellar environment, which can be quiescent or dominated by shocks and UV irradiation.

In Fig. 1 we display the spectrum toward the massive protostar NGC7538: IRS9. ISO allowed for the first time to observe the complete range between 2.5-200 $\mu \mathrm{m}$ without telluric contamination. Many features can be seen in the near and mid-infrared, which are discussed in detail in the following sections.

\section{Water $\left(\mathrm{H}_{2} \mathrm{O}\right)$}

Water ice showing main bands at $3.1,6.0$ and $13.5 \mu \mathrm{m}$ is the most abundant ice component in the interstellar medium as well as in the Solar System. Abundances of other species are therefore scaled relative to water ice. Water ice sublimates at $\sim 100 \mathrm{~K}$ under interstellar conditions and is therefore considered as the most refractory ice component. Highly volatile species such as $\mathrm{CO}, \mathrm{O}_{2}$ and $\mathrm{N}_{2}$ are sublimating already below $20 \mathrm{~K}$. This implies that layers with different ice composition are formed according to the temperature zones in protostellar regions. The profile of the water ice feature at $3.1 \mu \mathrm{m}$ can be used to derive temperature conditions in the vicinity of protostars (Smith et al. 1989). 


\section{Polycyclic Aromatic Hydrocarbons}

A weak absorption band at $3.25 \mu \mathrm{m}$ is detected in a number of massive YSO's (Brooke et al. 1996, 1999; Sellgren et al. 1994). It has been tentatively assigned to the $\mathrm{CH}$ stretching mode of frozen Polycyclic Aromatic Hydrocarbons (PAHs). A further tentative detection of the aromatic CC absorption feature at $6.2 \mu \mathrm{m}$ has been reported (Schutte et al. 1998). Certainly, it is expected that PAHs, an ubiquitous component of the interstellar medium, are embedded in the ices in dense clouds.

\section{Methanol $\left(\mathrm{CH}_{3} \mathrm{OH}\right)$}

Four features of solid methanol have been observed towards high mass young stellar objects: the $\nu_{3}$ band near $3.53 \mu \mathrm{m}\left(2830 \mathrm{~cm}^{-1}\right)$, the combination band around $3.87 \mu \mathrm{m}\left(2580 \mathrm{~cm}^{-1}\right)$, the $\nu_{7} / \nu_{11}$ feature near $8.8 \mu \mathrm{m}\left(1130 \mathrm{~cm}^{-1}\right)$, and the $\nu_{8}$ mode near $9.75 \mu \mathrm{m}\left(1030 \mathrm{~cm}^{-1}\right)$ (Grim et al. 1991; Allamandola et al. 1992; Schutte et al. 1996a; Skinner et al. 1992; Dartois et al. 1999a; Brooke et al. 1999). Of these the $\nu_{3}$ band is most widely observed, with detections so far toward 5 high mass YSO's (W33A, NGC7538:IRS9, GL2136, GL7009S, and MonR2 IRS2). Abundances vary widely, i.e., from $30 \%$ to $<2 \%$. There is a noticeable dichotomy in the methanol abundances for high and low mass YSO's. Observations of the latter class of objects have not yet given any detection, indicating a methanol abundance of $<6 \%$ (Chiar et al. 1996).

Detailed comparison with laboratory spectra indicates that methanol ice is not homogeneously mixed with the dominant $\mathrm{H}_{2} \mathrm{O}$ ice, but is contained in a separate, methanol-rich ice phase (Skinner et al. 1992; Dartois et al. 1999a; Ehrenfreund et al. 1999). Especially the $\nu_{8}$ mode is sensitive to the ice matrix. A good fit can be obtained by a mixture of $\mathrm{H}_{2} \mathrm{O}, \mathrm{CO}_{2}$ and $\mathrm{CH}_{3} \mathrm{OH}$ with similar abundances (Ehrenfreund et al. 1998, 1999).

\section{Deuterated Water (HDO)}

ISO spectra of the high-mass YSO's NGC7538:IRS9 and W33A show a weak feature at $4.1 \mu \mathrm{m}$. Teixera et al. (1999) showed that this feature can be reasonably matched with $\mathrm{HDO}$ diluted in $\mathrm{H}_{2} \mathrm{O}$ ice. This would indicate very high HDO abundances of $0.1-1 \%$ towards these objects. However, the $4.1 \mu \mathrm{m}$ band is very close to the SWS band transition $1 \mathrm{E} \rightarrow 2 \mathrm{~A}$ (de Graauw et al. 1996a). Therefore, an in-depth analysis of the limitations of the data reduction will be necessary to establish the reality of this band.

\section{Carbon Dioxide $\left(\mathrm{CO}_{2}\right)$}

The $\mathrm{CO}_{2}$ molecule is one of the major discoveries of ISO, visible in bands at $4.27,4.38$ and $15.2 \mu \mathrm{m}$ (de Graauw et al. 1996b). Though already seen in IRASLRS data (d'Hendecourt \& de Muizon 1989), a number of papers on $\mathrm{CO}_{2}$ have been published since the ISO launch (d'Hendecourt et al. 1996; Ehrenfreund et al. 1996; Gürtler et al. 1996; Gerakines et al. 1999). $\mathrm{CO}_{2}$ is responsible for 


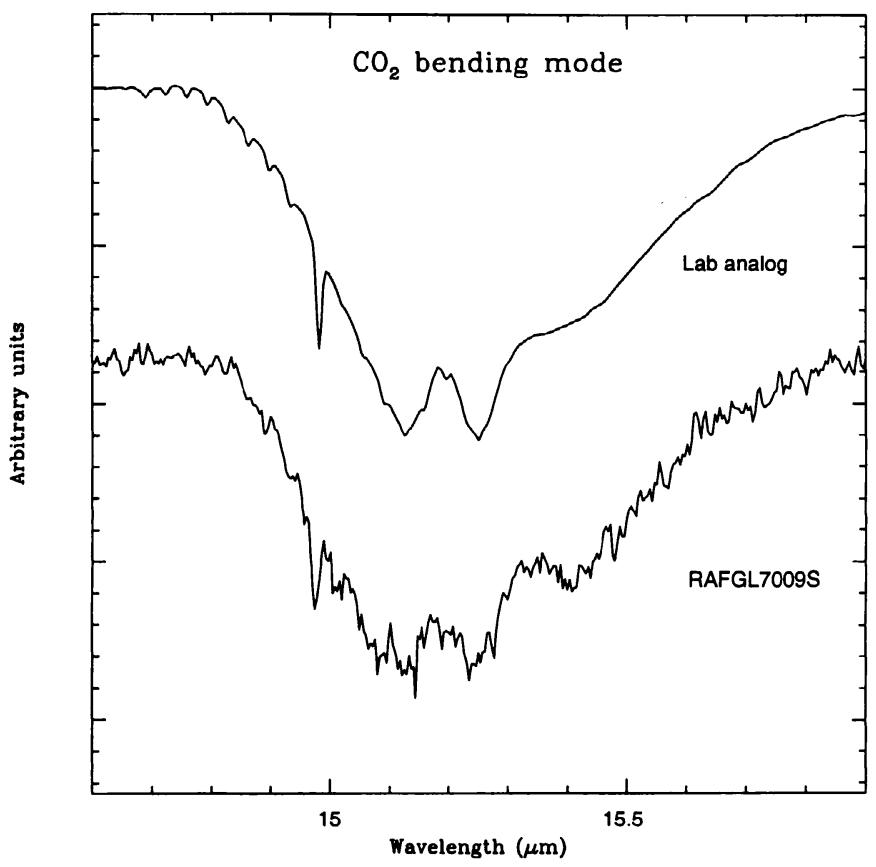

Figure 2. Comparison of a laboratory spectrum of the $\mathrm{CO}_{2}$ bending mode in a $\mathrm{H}_{2} \mathrm{O}: \mathrm{CH}_{3} \mathrm{OH}: \mathrm{CO}_{2}=1: 1: 1$ mixture with the ISO-SWS spectrum toward the protostar RAFGL7009S.

perturbations in the ice matrix, which can be traced in the infrared spectrum. This allows us to infer the ice composition, as well as temperature and irradiation conditions in the line of sight.

Fig. 2 shows a laboratory spectrum of the $\mathrm{CO}_{2}$ bending mode at $15.2 \mu \mathrm{m}$ in comparison with the infrared spectrum of the protostar RAFGL7009S. The match indicates that the $\mathrm{CO}_{2}$ ice must be "hot" and that interactions with $\mathrm{CH}_{3} \mathrm{OH}$ lead to intermolecular complexes (Lewis complexes) in the ice which show a signature at $15.4 \mu \mathrm{m}$ (Ehrenfreund et al. 1998; Dartois et al. 1999b). The peculiar shape of the $\mathrm{CO}_{2}$ bending mode is seen towards many protostellar objects (Gerakines et al. 1999) and indicates that ice segregation is an ubiquitous process in such environments (Ehrenfreund et al. 1998). The ${ }^{12} \mathrm{CO}_{2}$ stretch at $4.27 \mu \mathrm{m}$ has been also observed in field stars in the Taurus association, which are known to be quiescent in nature (Whittet et al. 1998). The $\mathrm{CO}_{2}$ formation in space remains a puzzle in interstellar chemistry. The abundance of $\mathrm{CO}_{2}$ relative to water seems relatively constant, namely $15 \%$ in various environments. The question remains if $\mathrm{CO}_{2}$ is formed by surface reactions (by oxidation of $\mathrm{CO}$ ) or by energetic processing (UV irradiation or cosmic rays). Whereas efficient formation of $\mathrm{CO}_{2}$ from photolysis of $\mathrm{H}_{2} \mathrm{O} / \mathrm{CO}$ ices is observed in the laboratory, the constant abundances of $\mathrm{CO}_{2}$ in different environments seem to point to another formation mechanism. The abundance of $\mathrm{CO}_{2}$ in the gas phase is surprisingly low and may be due to destruction of $\mathrm{CO}_{2}$ in shocks (Charnley \& Kaufman 
2000). The high abundances and strong transition of $\mathrm{CO}_{2}$, which show even saturation in some objects, allow accurate measurements of the ${ }^{13} \mathrm{CO}_{2}$ band. Laboratory fits with ${ }^{13} \mathrm{CO}_{2}$ observations confirm that $\mathrm{CO}_{2}$ seems to exist in different ice phases and that a high amount of $\mathrm{CO}_{2}$ is annealed (at higher temperature) (Boogert et al. 2000). Accurate measurements of $\mathrm{CO}_{2}$ with ISO-SWS allowed for the first time to measure the $\left.\left[{ }^{12} \mathrm{C}\right] /{ }^{13} \mathrm{C}\right]$ isotope ratio in protostellar regions (Boogert et al. 2000).

\section{7. $\mathrm{XCN} / \mathrm{The}$ Cyanate Ion $\left(\mathrm{OCN}^{-}\right)$}

A feature at $4.62 \mu \mathrm{m}$ is observed towards a large fraction of high- and low mass YSO's (Lacy et al. 1984; Tegler et al. 1995; Pendleton et al. 1999; d'Hendecourt et al. 1996). Its position is indicative of a nitrile or iso-nitrile, hence its common designation as the "XCN band". It was noticed early on that this band can be well matched by a laboratory feature produced by irradiation of ices containing $\mathrm{CO}$ and $\mathrm{NH}_{3}$ (Lacy et al. 1984). This feature is ascribed to the ion $\mathrm{OCN}^{-}$, which is thought to arise from acid-base reactions following photochemical production of iso-cyanic acid (HNCO: Grim \& Greenberg 1987; Schutte \& Greenberg 1997; Demyk et al. 1998). Thorough studies of alternative candidates like organic (iso-) nitriles showed that these molecules do not satisfy the observational constraints, i.e., they either do not provide a good match, or they show additional unobserved bands (Bernstein et al. 1995; Pendleton et al. 1999). We note however that it should not a-priori be assumed that the production mechanism of interstellar $\mathrm{OCN}^{-}$is the same as in the laboratory. For example, a more direct path may be possible, where $\mathrm{HNCO}$ is produced on the grain surface (Hasegawa et al. 1992), followed by formation of the ion through acid-base reactions. Laboratory studies are required to investigate the yield of such a mechanism.

\section{Carbon Monoxide (CO)}

The stretching mode of $\mathrm{CO}$ is widely observed towards high- and low-mass YSO's as well as field stars. It consists of two components, a broad feature (FWHM $\approx 12$ $\left.\mathrm{cm}^{-1}\right)$ centered at $4.67 \mu \mathrm{m}\left(2136 \mathrm{~cm}^{-1}\right)$, and a more narrow band $\left(3-11 \mathrm{~cm}^{-1}\right)$ near $4.68 \mu \mathrm{m}\left(2140 \mathrm{~cm}^{-1}\right)$. The broad component can be matched by CO diluted in a polar, $\mathrm{H}_{2} \mathrm{O}$-dominated, ice, while the narrower band is reproduced by $\mathrm{CO}$ in apolar matrices $\left(\mathrm{O}_{2}, \mathrm{~N}_{2}, \mathrm{CO}\right.$ itself: Sandford et al. 1988; Tielens et al. 1991; Chiar et al. 1998; Teixera et al. 1998). The abundance of the "apolar" CO ice is correlated with the overall ice temperature in the line of sight (Smith et al. 1989), and is generally considerably higher toward field stars and low mass YSO's than towards high-mass embedded objects (Kerr et al. 1993; Chiar et al. 1994,1995, 1998). On the other hand, the abundance of the "polar" CO component does not correlate with ice temperature indicators or environment. This is consistent with laboratory experiments, which show that a limited quantity of $\mathrm{CO}$ can become firmly embedded in an $\mathrm{H}_{2} \mathrm{O}$-dominated ice matrix, if both species condense simultaneously (Sandford \& Allamandola 1988). For low temperature line-of-sights, where thermal evaporation of CO is small, the apolar CO component dominates the polar component by a factor of $\sim 10$. The width of the apolar feature is quite variable. Generally, the most 
narrow features are observed for field stars, or low-mass YSO's with a high CO abundance, which are dominated by quiescent foreground material. The narrow features can be matched well with pure or almost pure $\mathrm{CO}$ ice, indicating that the apolar ice in the quiescent dense medium has a "simple" composition. The wider apolar bands indicate that more complex multi-component apolar ices are present in protostellar regions (Chiar et al. 1998).

\section{Carbonylsulfide (OCS)}

Carbonylsulfide (OCS) is currently the only sulphur-bearing species observed in interstellar ices. Its main feature at $4.9 \mu \mathrm{m}$ is seen toward $\sim 10$ objects (Palumbo et al. 1995, 1997). This band may be contaminated by $\mathrm{CH}_{3} \mathrm{OH}$ and $\mathrm{CO}_{3}$. Therefore the abundances are probably $20 \%$ lower than the current estimates of $0.05-0.18 \%$ OCS relative to water ice (Ehrenfreund et al. 1999).

\section{The 6-8 $\mu \mathrm{m}$ region}

The 6-8 $\mu \mathrm{m}$ region is dominated by two large absorption bands, the bending mode of water ice at $6 \mu \mathrm{m}$ and an unidentified band at $6.8 \mu \mathrm{m}$.

\subsection{Formaldehyde $\left(\mathrm{H}_{2} \mathrm{CO}\right)$}

An initial detection of the band of solid formaldehyde was reported from the observation of an absorption band at $3.47 \mu \mathrm{m}$ towards the high mass YSO GL2136 (Schutte et al. 1996a). However, this detection could not be confirmed by more recent observations (Brooke et al. 1999). ISO observations of RAFGL7009S and NGC7538:IRS9 however show a weak feature at $5.81 \mu \mathrm{m}$ superimposed on the $6 \mu \mathrm{m} \mathrm{H}_{2} \mathrm{O}$ band, which can likely be ascribed to the $\nu_{2}$ feature of solid $\mathrm{H}_{2} \mathrm{CO}$ (Ehrenfreund et al. 1997a; Keane et al. 2000). The corresponding abundance is a few percent relative to water ice.

\subsection{Formic acid (HCOOH)}

ISO gave the first high quality observations of the $6.0 \mu \mathrm{m}$ absorption band that is generally seen towards embedded objects. While it was previously thought that this band could be fully ascribed to the deformation mode of $\mathrm{H}_{2} \mathrm{O}$, the new data clearly show excess absorption on the blue as well as the red wing of this feature (Schutte et al. 1996b; Keane et al. 2000). This shows that at least two additional components are present. Addition of the CO stretching mode of organic acids, in particular formic acid $(\mathrm{HCOOH})$, to the water feature produced a satisfactory match of the blue wing of the spectrum of NGC7538:IRS9 (Schutte et al. 1996b). The derived abundance is $\sim 3 \%$ relative to water ice. The red excess is possibly caused by the $6.2 \mu \mathrm{m} \mathrm{C}-\mathrm{C}$ stretching mode of solid aromatic moieties (see §3).

\subsection{Molecular oxygen $\left(\mathrm{O}_{2}\right)$}

The oxygen budget in interstellar clouds has been an important subject, since $\mathrm{O}$ is the most abundant species after $\mathrm{H}$ and He. Though recent HST observations indicate a lower oxygen abundance of only $2 / 3$ in the interstellar medium 
compared to our Solar System, a large amount of oxygen can not be readily observed. It has been argued that large amounts of oxygen could be hidden in molecular form in interstellar gas and ice. Indirect measurements of $\mathrm{O}_{2}$ provide only small upper limits (Marechal et al. 1997). The SWAS satellite confirmed that $\mathrm{O}_{2}$ is a minor component in the interstellar gas. The search for solid $\mathrm{O}_{2}$ at $6.4 \mu \mathrm{m}$ (a weak infrared active transition visible only in the solid state) and the deconvolution of $\mathrm{CO}$ profiles of dense cloud sources led to an upper limit of $6 \%$ of $\mathrm{O}_{2}$ ice in the total oxygen budget (Vandenbussche et al. 1999). Most of the oxygen is likely in atomic form and resides in the interstellar gas.

\subsection{The $6.8 \mu \mathrm{m}$ band}

A strong absorption feature near $6.8 \mu \mathrm{m}$ is generally observed toward massive protostars. Since the feature falls in the region of telluric $\mathrm{H}_{2} \mathrm{O}$, the first highquality observations had to await the launch of ISO. The new data show that this band shows a very strong shift from object to object (Keane et al. 2000). No plausible candidates have yet been put forward (Schutte et al. 1996b; Schutte 1999; Keane et al. 2000). While the $\mathrm{CH}$ deformation mode of organic molecules fall in this region, other features of such species are lacking in the observations. It is expected that the peculiar behaviour of the $6.8 \mu \mathrm{m}$ band will pose strong constraints on the nature of its carrier.

\section{The $7.24 \& 7.41 \mu \mathrm{m}$ Weak Bands}

Two weak absorption features at 7.24 and $7.41 \mu \mathrm{m}$ are present in the spectrum of the high-mass YSO W33A. These features fall in the $\mathrm{CH}$ deformation region of organic molecules, leaving numerous potential carriers. A number of candidates were selected by Schutte et al. (1999) on the basis of a comparison with standard infrared spectroscopic libraries, and were spectroscopically analyzed after being embedded in low-temperature astrophysical ice analogs. Of these, formic acid $(\mathrm{HCOOH})$, acetaldehyde $\left(\mathrm{CH}_{3} \mathrm{HCO}\right)$ and the formate ion $\left(\mathrm{HCOO}^{-}\right.$, obtained from acid-base reactions involving formic acid) gave the best spectral matches to the features. Abundances of $0.3-3 \%$ relative to water ice were indicated. Further analysis of the ISO data in the near future will allow better abundance constraints, since these molecules all have strong features between 5.5 and 6.5 $\mu \mathrm{m}$.

\section{Methane $\left(\mathrm{CH}_{4}\right)$}

So far, ISO detected the $\nu_{4}$ feature of solid methane at $7.68 \mu \mathrm{m}$ for three high mass YSO's: W33A, RAFGL7009S, and NGC7538:IRS9 (Boogert et al. 1996, 1998; Dartois et al. 1998). The abundance varies between $1.3-4 \%$. The profile of the feature shows that methane is embedded in a polar ice matrix, consisting of $\mathrm{H}_{2} \mathrm{O}$ and possibly $\mathrm{CH}_{3} \mathrm{OH}$. 


\section{Ammonia $\left(\mathrm{NH}_{3}\right)$}

The detection of solid $\mathrm{NH}_{3}$ is difficult, since its three mid-infrared features, the $\nu_{1} / \nu_{3}$ at $2.96 \mu \mathrm{m}$, the $\nu_{4}$ at $6.16 \mu \mathrm{m}$ and $\nu_{2}$ at $9.35 \mu \mathrm{m}$ all blend with much stronger absorption of either $\mathrm{H}_{2} \mathrm{O}$ ice or silicates. Recently, a first detection of the umbrella mode was reported (Lacy et al. 1998). While the abundance deduced from this detection is $\sim 13 \%$ (obtained with an updated version of the $\mathrm{NH}_{3}$ band strength: Kerkhof et al. 1999), the general absence of the $2.96 \mu \mathrm{m}$ feature in the spectra of YSO's (e.g. Smith et al. 1989; Brooke et al. 1999) is interpreted to give an upper limit of $\sim 10 \%$ relative to $\mathrm{H}_{2} \mathrm{O}$. However, the stringency of the upper limit imposed by the missing $2.96 \mu \mathrm{m}$ is still unclear. It should for example be noted that Mie calculations of the spectrum of a silicate core coated with a $\mathrm{H}_{2} \mathrm{O}: \mathrm{NH}_{3}=4: 1$ ice only give a very weak $2.96 \mu \mathrm{m}$ feature (Smith et al. 1989). We conclude that, while the $\mathrm{NH}_{3}$ abundance should be lower than $\sim 20 \%$ in general, better constraints await further observations and modelling of the $\nu_{1} / \nu_{3}$ and $\nu_{2}$ features.

Table 1. Millenium abundances of interstellar ices.

\begin{tabular}{llll}
\hline & $\begin{array}{l}\text { NGC7538:IRS9 } \\
\text { high-mass protostar }\end{array}$ & $\begin{array}{l}\text { Elias 29 } \\
\text { low-mass protostar }\end{array}$ & $\begin{array}{l}\text { Elias 16 } \\
\text { field star }\end{array}$ \\
\hline $\mathrm{H}_{2} \mathrm{O}$ & 100 & 100 & 100 \\
$\mathrm{CO}$ & 2 (polar) & 0.8 & 7 (polar) \\
& 14 (apolar) & 4.8 & 27 (apolar) \\
$\mathrm{CO}_{2}$ & 20 & 22 & 15 \\
$\mathrm{CH}_{4}$ & 2 & $<1.6$ & - \\
$\mathrm{CH}_{3} \mathrm{OH}$ & 5 & $<4$ & $<3.4$ \\
$\mathrm{H}_{2} \mathrm{CO}$ & 2 & - & - \\
$\mathrm{OCS}$ & 0.05 & $<0.08$ & - \\
$\mathrm{NH}_{3}$ & 13 & $<9.2$ & $<6$ \\
$\mathrm{C}_{2} \mathrm{H}_{6}$ & $<0.4$ & - & - \\
$\mathrm{HCOOH}$ & 3 & - & - \\
$\mathrm{O}_{2}$ & $<20$ & - & - \\
$\mathrm{OCN}^{-}$ & 1 & $<0.24$ & $<0.4$ \\
\hline
\end{tabular}

\section{Discussion}

After ISO, the composition of interstellar ices in various environments can be derived from the spectroscopic record with unprecedented detail. One result that stands out is the distinct difference between the composition of ices in the three classes of dense environments that are generally distinguished: High-mass YSO's, Low-mass YSO's and the general quiescent dense medium. Current abundances in such targets are listed in Table 1.

Ices near high mass YSO's are characterized by high abundances of methanol and $\mathrm{CO}_{2}$ mixed with a comparatively small amount of $\mathrm{H}_{2} \mathrm{O}$. Further out a transi- 


\section{THE CYCLE OF ICE AND GAS IN DENSE CLOUDS}

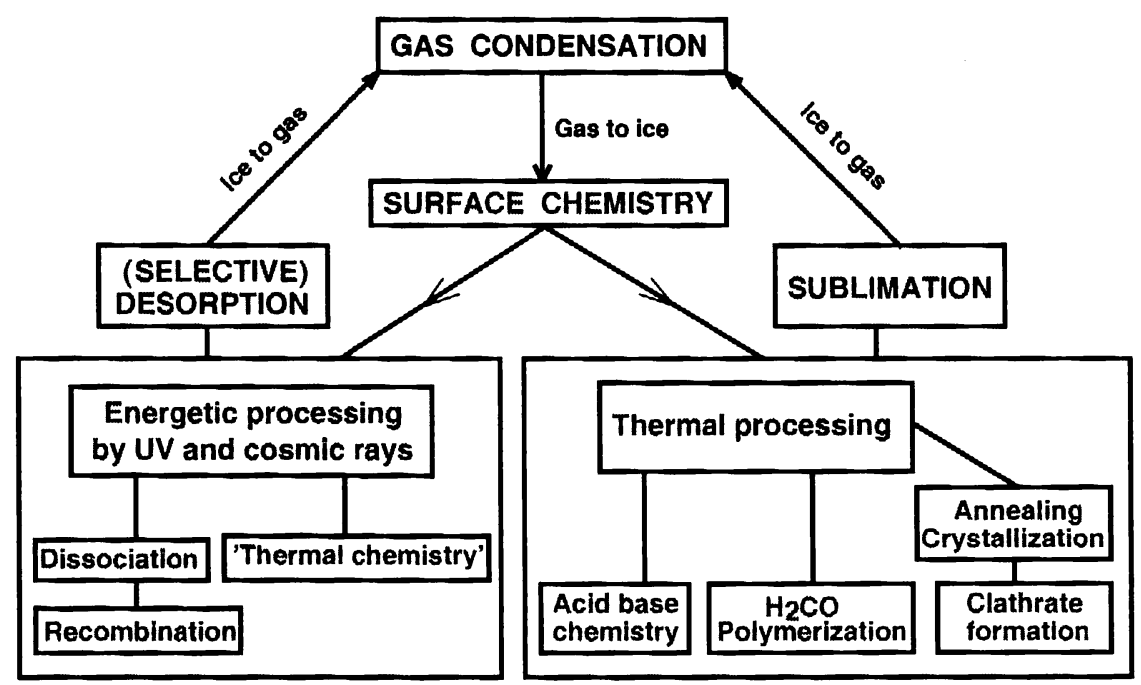

Figure 3. The evolution of ices in dense clouds.

tion occurs to more dense cloud-like ices, dominated by $\mathrm{H}_{2} \mathrm{O}$ with $\mathrm{CO}_{2}$ and some CO. Ices near low-mass YSO's contain less methanol, while also little methanol is present in ices in the quiescent medium. On the other hand, $\mathrm{CO}_{2}$ is ubiquitous in all dense environments. $\mathrm{OCN}^{-}$is present in both classes of YSO's, but seems underabundant in the quiescent medium. Also, the apolar ice component shows distinct variations; while consisting of almost pure $\mathrm{CO}$ in the quiescent medium (although an important inclusion of $\mathrm{N}_{2}$ cannot be excluded, due to its inert spectroscopic behaviour: Ehrenfreund et al. 1997b; Schutte 1999), its composition is considerably more complex in the environments of low- and high-mass YSO's.

The present observational constraints thus indicate that interstellar ices are severely re-processed in regions of star-formation. Obviously, besides "mild" processes like heating of the ice near the protostar, "violent" processes could also be of importance. These could be shocks originating from intense stellar winds, high fluxes of energetic particles, X-ray and UV, or even lightning (Bergin et al. 1999; Desch \& Cuzzi 1999; Teixera et al. 1998; Spaans et al. 1995). Each of these conditions can influence the chemistry in a variety of ways. For example, a high particle and/or UV field could highly ionize and atomize species in the gas phase and induce new chemical pathways. Upon condensation, these new products are modified by reactions with atoms on the grain surface. Finally, the ices can be processed directly by the cosmic ray particles and UV irradiation.

A schematic outline of processes which lead to the chemical modification of interstellar ices is given in Fig. 3. For a detailed understanding we refer to Schutte et al. 1993 (formaldehyde polymerization), Gerakines et al. 1996, Bernstein et al. 1995, Briggs et al. 1992, Grim et al. 1991 (UV processing), Ehrenfreund et al. 1999 (clathrate formation), Moore \& Hudson 1992, 1998, 
Palumbo et al. 1999 (cosmic ray processing), Schutte et al. 1999 (acid-base reactions), Léger et al. 1985 (selective desorption), Sandford \& Allamandola 1993 (sublimation).

Further experimental and theoretical effort is necessary to understand both the gas and grain chemistry of the peculiar conditions in star-forming regions. However, after ISO the observational constraints are quite extensive, and we may therefore hope that such a broad and thorough approach will in the end greatly advance our knowledge of the physics and chemistry of star formation.

\section{References}

Allamandola, L.J., Sandford, S.A., Tielens, A.G.G.M., \& Herbst, T.M. 1992, ApJ, 399, 134

Bergin, E.A., Neufeld, D.A., \& Melnick, G.J. 1999, ApJ, 510, L145

Bernstein, M.P., Sandford, S.A, Allamandola, L.J., Chang S., \& Scharberg, M.A. 1995, ApJ, 454, 327

Boogert, A.C.A., Schutte, W.A., Tielens, A.G.G.M., Whittet, D.C.B., Helmich, F.P., Ehrenfreund, P., Wesselius, P.R., de Graauw, Th., \& Prusti, T. 1996, A\&A, 315, L377

Boogert, A.C.A., Helmich, F.P., van Dishoeck, E.F., Schutte, W.A., Tielens, A.G.G.M., \& Whittet, D.C.B. 1998, A\&A, 336, 352

Boogert, A.C.A., Ehrenfreund, P., Gerakines, P.A., et al. 2000, A\&A, 353, 349

Briggs, R., Ertem, G., Ferris, J.P., et al. 1992, Origins of Life and Evolution of the Biosphere, 22, 287

Brooke, T.Y., Sellgren, K., \& Smith, R.G. 1996, ApJ, 459, 209

Brooke, T.Y., Sellgren, K., \& Geballe, T.R. 1999, ApJ, 517, 883

Charnley, S.B. \& Kaufman, M.J. 2000, ApJ, 529, L111

Chiar, J.E., Adamson, A.J., Kerr, T.H., \& Whittet, D.C.B. 1994, ApJ, 426, 240 1995, ApJ, 455, 234

Chiar, J.E., Adamson, A.J., \& Whittet, D.C.B. 1996, ApJ, 472, 665

Chiar, J.E., Gerakines, P.A., Whittet, D.C.B., Pendleton, Y.J., Tielens, A.G.G.M., Adamson, A.J., \& Boogert, A.C.A. 1998, ApJ, 498, 716

Dartois, E., d'Hendecourt, L., Boulanger, F., Jourdain de Muizon, M., Breitfellner, M., Puget, J.-L., \& Habing, H.J. 1998, A\&A, 331, 651

Dartois E., Schutte, W.A., Geballe, T.R., Demyk, K., Ehrenfreund, P., \& d'Hendecourt, L. 1999a, A\&A, 342, L32

Dartois E., Demyk, K., d'Hendecourt, L., \& Ehrenfreund, P. 1999b, A\&A, 351, 1066

de Graauw, Th., Haser, L.N., Beintema, D.A., et al. 1996a, A\&A, 315, L49

de Graauw, Th., Whittet, D.C.B., Gerakines, P.A. 1996b, A\&A, 315, L345

Demyk, K., Dartois, E., d'Hendecourt, L., Jourdain de Muizon, M., Heras, A.M., \& Breitfellner, M. 1998, A\&A, 339, 553

Desch, S.D. \& Cuzzi, J.N. 1999, Icarus, submitted

d'Hendecourt, L. \& Jourdain de Muizon, M. 1989, A\&A, 223, L5

d'Hendecourt, L., Jourdain de Muizon, M., Dartois, E., et al. 1996, A\&A, 315, L365

Ehrenfreund, P., Boogert, A.C.A., Gerakines, P.A., et al. 1996, A\&A, 315, L341

Ehrenfreund, P., Boogert, A.C.A., Gerakines, P.A., et al. 1997b, A\&A, 328, 649

Ehrenfreund, P., d'Hendecourt, L., Dartois, E., et al. 1997a, Icarus, 130, 1 
Ehrenfreund, P., Dartois, E., Demyk, K., \& d'Hendecourt, L. 1998, A\&A, 339, L17

Ehrenfreund, P., Kerkhof, O., Schutte, W.A., et al. 1999, A\&A, 350, 240

Gerakines, P.A., Schutte, W.A., Ehrenfreund, P., \& van Dishoeck, E.F. 1996, A\&A, 312, 289

Gerakines, P.A., Whittet, D.C.B., Ehrenfreund, P., et al. 1999, ApJ, 522, 357

Grim, R.J.A. \& Greenberg, J.M., 1987, ApJ, 321, L91

Grim, R.J.A., Baas, F., Geballe, T.R., Greenberg, J.M., \& Schutte, W. 1991, A\&A, 243, 473

Gürtler, J., Henning, Th., Koempe, C., et al. 1996 A\&A, 315, L189

Hasegawa, T.I., Herbst, E., \& Leung, C.M. 1992, ApJS, 82, 167

Keane, J., et al. 2000, A\&A, submitted

Kerkhof, O., Schutte, W.A., \& Ehrenfreund, P. 1999, A\&A, 346, 990

Kerr, T.H., Adamson, A.J., \& Whittet, D.C.B. 1993, MNRAS, 262, 1047

Lacy, J.H., Baas, F., Allamandola, L.J., et al. 1984, ApJ, 276, 53

Lacy, J.H., Faraji, H., Sandford, S.A., \& Allamandola, L.J. 1998, ApJ, 501, L105

Léger, A., Jura, M., \& Omont, A. 1985, A\&A, 144, 147

Marechal, P., Viala, Y.P., \& Pagani, L. 1997, A\&A, 328, 617

Moore, M.H. \& Hudson, R.L. 1992, ApJ, 401, 353

1998, Icarus, 135, 518

Palumbo, M.E., Tielens, A.G.G.M., \& Tokunaga, A.T. 1995, ApJ, 449, 674

Palumbo, M.E., Geballe, T.R., Tielens, A.G.G.M., et al. 1997, ApJ, 479, L839

Palumbo, M.E., Castorina, A.C., \& Strazzulla, G. 1999, A\&A, 342, 551

Pendleton, Y.J., Tielens, A.G.G.M., Tokunaga, A.T., \& Bernstein, M.P. 1999, ApJ, 513, 294

Sandford, S.A., Allamandola, L.J., Tielens, A.G.G.M., \& Valero, L.J. 1988, ApJ, 329,498

Sandford, S.A. \& Allamandola, L.J. 1988, Icarus, 76, 201

1993, ApJ, 417, 815

Schutte, W.A., Allamandola, L.J., \& Sandford, S.A. 1993, Icarus, 104, 118

Schutte, W.A., Gerakines, P.A., Geballe, T.R., van Dishoeck, E.F., \& Greenberg, J.M. 1996a, A\&A, 309, 633

Schutte, W.A., Tielens, A.G.G.M., Whittet, D.C.B., et al. 1996b, A\&A, 315, L333

Schutte, W.A. \& Greenberg, J.M. 1997, A\&A, 317, L43

Schutte, W.A., Van der Hucht, K.A., Whittet, D.C.B., et al. 1998, A\&A, 337, 261

Schutte, W.A. 1999, in Laboratory Astrophysics and Space Research, eds. P. Ehrenfreund et al. Kluwer Academic Publisher, 69

Schutte, W.A., Boogert, A.C.A., Tielens, A.G.G.M., et al. 1999, A\&A, 343, 966

Sellgren, K., Smith, R.G., \& Brooke, T.Y. 1994, ApJ, 433, 179

Skinner, C.J., Tielens, A.G.G.M., Barlow, M.J., \& Justtanont, K. 1992, ApJ, 399, L79

Smith, R.G., Sellgren, K., \& Tokunaga, A.T. 1989, ApJ, 344, 413

Spaans, M., Hogerheijde, M.R., Mundy, L.G., \& van Dishoeck, E.F. 1995, ApJ, $455, \mathrm{~L} 167$

Tegler, S.C., Weintraub, D.A., Rettig, T.W., Pendleton, Y.J., Whittet, D.C.B., \& Kulesa, C.A. 1995, ApJ, 439, 279

Teixera, T.C., Emerson, J.P., \& Palumbo, M.E. 1998, A\&A, 330, 711

Teixera, T.C., Devlin, J.P., Buch, V., \& Emerson, J.P. 1999, A\&A, 347, L19 
Tielens, A.G.G.M., Tokunaga, A.T., Geballe, T.R., \& Baas, F. 1991, ApJ, 381, 181

Whittet, D.C.B., Schutte, W.A., Tielens, A.G.G.M., et al. 1996, A\&A, 315, L357.

Whittet, D.C.B., Gerakines, P.A., Tielens, A.G.G.M., et al. 1998, ApJ, 498, L159.

Vandenbussche, B., Ehrenfreund, P., Boogert, A.C.A., et al. 1999, A\&A, 346, L54

\section{Discussion}

J. H. Black: Please comment on the observation of solid $\mathrm{H}_{2}$.

$P$. Ehrenfreund: $\mathrm{H}_{2}$ has no active IR transition since it is a homonuclear molecule. In the solid state, however, the symmetry is broken and weak infrared active transitions are possible. Laboratory data allowed a search for $\mathrm{H}_{2}$ in the NIR in dense clouds. A feature has been identified in WL 5 which has been attributed to solid $\mathrm{H}_{2}$ by Sandford et al. (1993, Science, 262, 400).

T. Geballe: Comment: I and the other authors of the discovery paper are retracting it. The feature originally reported as solid $\mathrm{H}_{2}$ is fully explained by a band of $\mathrm{CO}$ in the background star (WL 5).

T. Geballe: A $\mathrm{N}_{2}$ ice band at $2.15 \mu \mathrm{m}$ has been detected on the surfaces of Pluto and Triton. Perhaps it will be usable in some circumstances to search for interstellar $\mathrm{N}_{2}$ ice.

P. Ehrenfreund: The fundamental transition of solid $\mathrm{N}_{2}$, which is weakly IR active falls at $4.28 \mu \mathrm{m}$. Although large amounts of $\mathrm{N}_{2}$ ice cannot be excluded on grains in dense clouds the detection of solid $\mathrm{N}_{2}$ will remain extremely difficult. The weak $4.28 \mu \mathrm{m}$ feature of $\mathrm{N}_{2}$ ice falls adjacent to the large $\mathrm{CO}_{2}$ ice band and will in most cases be buried in the red wing of this band. Additionally the isotopic band of $\mathrm{C}^{18} \mathrm{O}^{16} \mathrm{O}$ overlaps with the $\mathrm{N}_{2}$ transition. The ovetone band in the very near-IR may be too weak to be detected in the ISM.

C. $Q i$ : Can you comment on any observations of clathrate hydrates in the ISM ices? How about in solar system objects?

$P$. Ehrenfreund: In the solar system, clathrates have been observed. Until now there was no evidence for clatherate hydrates in the interstellar medium. ISO showed that extensive ice segregation occurs in the vicinity of protostars. From ISO IR spectra of such regions we can conclude that close to the protostars ices may partly be crystallized and $\mathrm{CH}_{3} \mathrm{OH}$ or $\mathrm{CO}_{2}$ might be trapped in clathrate hydrates. 